\title{
Adaptive fractional PID control of biped robots with time-delayed feedback
}

\author{
IEEE MEMBER Joel Perez P*, Jose P. Perez, Mayra Flores Guerrero, Ruben Perez P. , \\ Oscar Rangel Aguilar, Daniel Ramirez-V, Joey Perez F., and J. Javier Perez D.
}

Department of Physical and Mathematical Sciences, Autonomus University of Nuevo Leon (FCFM,UANL), Cd. Universitaria, San Nicolas de los Garza, Nuevo Leon, 66450, Mexico

\begin{abstract}
This paper presents the application of Fractional Order TimeDelay adaptive neural networks to the trajectory tracking for chaos synchronization between Fractional Order delayed plant, reference and Fractional Order Time-Delay adaptive neural networks. The proposed new control scheme is applied via simulations to control of a 4-DOF Biped Robot [1]. The main methodologies, on which the approach is based, are Fractional Order PID the Fractional Order Lyapunov-Krasovskii functions methodology. The structure of the biped robot is designed with two degrees of freedom per leg, corresponding to the knee and hip joints. Since torso and ankle are not considered, it is obtained a 4-DOF system, and each leg, we try to force this biped robot to track a reference signal given by undamped Duffing equation. The tracking error is globally asymptotically stabilized by two control laws derived based on a Lyapunov-Krasovski functional.
\end{abstract}

\section{Introduction}

Fractional calculus is a generalization of differential and integral calculus which involves generalized functions. The first to work this new branch of mathematics was Leibniz. Due to the growing interest in the applications of fractional calculation, in this work we obtain conditions that guarantee the tracking of trajectories of nonlinear systems generated by differential equations of fractional order which we will call plants (This term is widely used in engineering), which in our case will be a mechanical arm, a helicopter, a plane or limbs of a humanoid, all of fractional order.

The problem of tracking control of trajectories is very important, since the control function allows the non-linear system to carry out a previously assigned task, work or trajectory, for example, a mechanical arm and its objective is to cut a piece with a previously generated form, or the coupling of two aircraft in space. We include mathematical models with time delay, since the processing and transmission of information is important in this type of systems, which depending on the delay, these systems can generate undesirable oscillatory or chaotic dynamics, and cause instability in the mathematical model that describes the trajectory tracking error.

\footnotetext{
* Corresponding author: joel.perezpd@uanl.edu.mx
} 
The chapter is organized as follows: first, the general mathematical model of non-linear systems is proposed, as a second part, the Neural Network is proposed that will adapt to the non-linear system and the reference signal that both must follow, as a third part obtains the dynamics of the tracking error between the non-linear system and the reference, after obtaining conditions in the laws of adaptation of weights in the Neural Network and obtaining the control law that guarantees that the tracking error converges to zero, so that the non-linear system will follow the indicated reference signal, which is what was wanted to be demonstrated. Finally simulations are presented, which illustrate the theoretical results previously demonstrated.

There are several ways to define the fractional calculation, in this research we will use the well-known derivative of Caputo, which has equation (1):

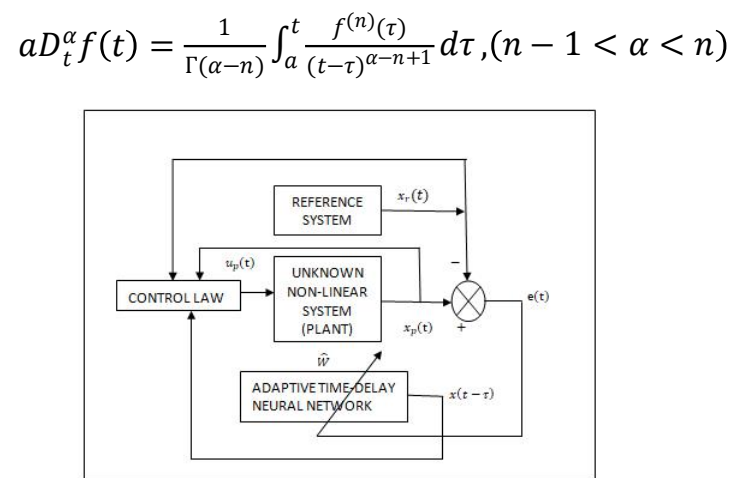

Fig. 1. Adaptive recurrent control diagram.

We use the scheme of figure 1 to indicate the procedure used in the obtaining of the laws of adaptation of weights and the laws of control that guarantee that the tracking error between the non-linear system, the neural network and the reference signal converges to zero.

\section{Time-delay adaptive neural network and the reference}

The nonlinear system, equation (5), which is forced to follow a reference signal:

$$
\begin{aligned}
& \quad \boldsymbol{a} \boldsymbol{D}_{\boldsymbol{t}}^{\boldsymbol{\alpha}} \boldsymbol{x}_{\boldsymbol{p}}=\boldsymbol{f}_{\boldsymbol{p}}\left(\mathbf{t}, \boldsymbol{x}_{\boldsymbol{p}}(\mathbf{t})+\boldsymbol{x}_{\boldsymbol{p}}(\mathbf{t}-\boldsymbol{\tau})\right), \mathbf{t} \in[\mathbf{0}, \mathbf{T}], \mathbf{0}<\boldsymbol{\alpha} \leq \mathbf{1}, \\
& x_{p}(\mathrm{t})=\mathrm{g}(\mathrm{t}), \text { where } x_{p}, f_{p} \in \mathfrak{R}^{n}, u \in \mathfrak{R}^{m}, g_{p} \in \mathfrak{R}^{\text {nxn }} .
\end{aligned}
$$

The differential equation will be modeled by the neural network [2]: $a D_{t}^{\alpha} x_{p}=\mathrm{A}(x)+$ $\mathrm{W}^{*} \Gamma_{\mathrm{z}}\left(x(t-\tau)+\Omega_{\mathrm{u}}\right.$

The tracking error between these two systems:

$$
w_{\text {per }}=x-x_{p} \text { and } a D_{t}^{\alpha} w_{\text {per }}=-k w_{\text {per }}
$$

We use the next hypotheses. The nonlinear system is [3]:

$$
a D_{t}^{\alpha} x_{p}=a D_{t}^{\alpha} x+w_{p e r}=\mathrm{A}(x)+\mathrm{W}^{*} \Gamma_{\mathrm{z}}[x(t-\tau)]+w_{p e r}+\Omega_{\mathrm{u}}
$$

where the $\mathrm{W}^{*}$ is the matrix weights.

\section{Tracking error problem}

In this part, we will analyze the trajectory tracking problem generated by

$$
a D_{t}^{\alpha} x_{r}=f_{r}\left(x_{r}, u_{r}\right), w_{r}, x_{r} \in \mathfrak{R}^{n}
$$

The time derivative of the error is:

$$
a D_{t}^{\alpha} e_{n}=a D_{t}^{\alpha} x-a D_{t}^{\alpha} x_{r}=\mathrm{A}(x)+\mathrm{W}^{*} \Gamma_{\mathrm{z}}[x(t-\tau)]+w_{p e r}+\Omega_{\mathrm{u}}-f_{r}\left(x_{r}, u_{r}\right)
$$


The equation (6), can be rewritten as follows, and the unknown plant will follow the fractional order reference signal, if:

$$
\begin{gathered}
a D_{t}^{\alpha} e=\mathrm{A} e+\mathrm{W}^{*} \Gamma_{\mathrm{z}}(x(t-\tau))-\widehat{W} \Gamma_{\mathrm{z}}\left(x_{r}(t-\tau)\right)-\mathrm{A} e+(A+I)\left(x-x_{r}\right)+ \\
\Omega\left(u-\alpha_{r}(\mathrm{t}, \widehat{W})\right)
\end{gathered}
$$

Now, $\widehat{W}$ is part of the approach, given by $W^{*}$. The Eq. (7), can be expressed as Eq. (8)

$$
\begin{gathered}
a D_{t}^{\alpha} e=\mathrm{A} e+\left(\mathrm{W}^{*}-\widehat{W}\right) \Gamma_{\mathrm{z}}(x(t-\tau))+\widehat{W} \Gamma\left(z(x(t-\tau))-z\left(x_{r}(t-\tau)\right)\right)+ \\
(A+I)\left(x-x_{r}\right)-\mathrm{A} e+\Omega\left(u-\alpha_{r}(\mathrm{t}, \widehat{W})\right) \\
\widetilde{W}=\mathrm{W}^{*}-\widehat{W} \text { and } \widetilde{u}=u-\alpha_{r}(\mathrm{t}, \widehat{W})
\end{gathered}
$$

And by replacing Eq. (9) in Eq. (8), we have:

$$
a D_{t}^{\alpha} e=(A+I) e+\widetilde{W} \sigma(x(t-\tau))+\widehat{W} \emptyset_{\sigma}(t-\tau)+\Omega u_{2}
$$

The control law, we will obtain using the fractional order Lyapunov-Krasovskii methodology.

\section{Study of trajectory tracking error}

Our mathematical model of the dynamics in the tracking error is described in (10). In this equation we can see that an equilibrium state of this system is $(e, \widehat{W})=0$.

Without loss of generality we can assume that the matrix $A$ is given $A=-\lambda I, \lambda>0$, where $I$ is the identity matrix of order $n x n$.

For the study of the stability of the tracking error we propose the following PID control law [4], widely used in science and engineering.

We will determine conditions in the parameters that guarantee that the tracking error converges to zero, and we will also use the following control law [5].

$$
\Omega u_{2}=K_{p} e+K_{i} a D_{t}^{-\alpha} e+K_{v} a D_{t}^{\alpha} e-\gamma\left(\frac{1}{2}+\frac{1}{2}\|\widehat{W}\|^{2} L_{\phi}^{2}\right) e
$$

We also include the following control law, $P I^{\lambda} D^{\alpha}[6]: u(t)=K_{p} e(t)+K_{i} a D_{t}^{-\lambda} e(t)+$ $K_{d} a D_{t}^{\alpha} e(t)$,

Substituting Eq. (11) in Eq. (10):

$$
\begin{aligned}
& a D_{t}^{\alpha} e=\frac{-1}{a}\left(\lambda-1+K_{p}\right) e+\frac{1}{a} \widetilde{W} \sigma(x(t-\tau))+\frac{1}{a} \widehat{W} \emptyset_{\sigma}(t-\tau)+ \\
& \frac{1}{a} K_{i} a D_{t}^{-\alpha} e-\frac{\gamma}{a}\left(\frac{1}{2}+\frac{1}{2}\|\widehat{W}\|^{2} L_{\phi}^{2}\right) e
\end{aligned}
$$

And if $w=\frac{1}{a} K_{i} a D_{t}^{-\alpha} e$, then $a D_{t}^{\alpha} w=\frac{1}{a} K_{i} e(t)$, [7], then Eq. (12) we rewrite as:

$$
\begin{gathered}
a D_{t}^{\alpha} e_{n}=\frac{-1}{a}\left(\lambda-1+K_{p}\right) e+\frac{1}{a} \widetilde{W} \sigma(x(t-\tau))+\frac{1}{a} \widehat{W} \emptyset_{\sigma}(t-\tau)+ \\
w-\frac{\gamma}{a}\left(\frac{1}{2}+\frac{1}{2}\|\widehat{W}\|^{2} L_{\phi}^{2}\right) e
\end{gathered}
$$

Let $V$ be, the next candidate Lyapunov function as [8] and [9] :

$$
V=\frac{1}{2}\left(e_{n}{ }^{T}, w^{T}\right)\left(e_{n}, w\right)^{T}+\frac{1}{2 a} \operatorname{tr}\left\{\widetilde{W}^{T} \widetilde{W}\right\}+\frac{1}{a} \int_{t-\tau}^{t}\left[\emptyset_{\sigma}^{T}(s) \widehat{W}^{T} \widehat{W} \emptyset_{\sigma}(s)\right] d s
$$

The fractional order time derivative of (14) along the trajectories of Eq. (13), and we select the next learning law from the neural network weights as in [10] and [11]:

Then Eq. (13) is reduced to

$$
\operatorname{tr}\left\{a D_{t}^{\alpha} \widetilde{W}^{T} \widetilde{W}\right\}=-e^{T} \widetilde{W} \sigma(x(t-\tau))
$$

$$
\begin{gathered}
a D_{t}^{\alpha} V=\frac{-1}{a}\left(\lambda-1+K_{p}\right) e^{T} e+\frac{e^{T}}{a} \widehat{W} \emptyset_{\sigma}(t-\tau)+\left(1+\frac{K_{i}}{a}\right) e^{T} w-\frac{\gamma}{a}\left(\frac{1}{2}+\frac{1}{2}\|\widehat{W}\|^{2} L_{\phi}^{2}\right) e^{T} e \\
+\quad \frac{1}{a}\left[\emptyset_{\sigma}^{T}(t) \widehat{W}^{T} \widehat{W} \emptyset_{\sigma}(t)-\emptyset_{\sigma}^{T}(t-\tau) \widehat{W}^{T} \widehat{W} \emptyset_{\sigma}(t-\tau)\right]
\end{gathered}
$$

Next, let's consider the following inequality proved in [12]

$$
X^{T} Y+Y^{T} X \leq X^{T} \Lambda X+Y^{T} \Lambda^{-1} Y
$$


Which holds for all matrices $X, Y \in \mathfrak{R}^{n x k}$ and $\Lambda \in \mathfrak{R}^{n x n}$ with $\Lambda=\Lambda^{T}>0$. Applying (22) with $\Lambda=I$ to the term $\frac{e^{T}}{a} \widehat{W} \emptyset_{\sigma}(t-\tau)$ from Eq. (16), where, we get

Here, we select $\left(1+\frac{K_{i}}{a}\right)=0$ and $K_{v}=K_{i}+1$, with $K_{v} \geq 0$ then $K_{i} \geq-1$, with this selection of the parameters from Eq. (16) is reduced to:

$$
a D_{t}^{\alpha} V \leq \frac{-1}{a}\left(\lambda-1+K_{p}\right) e^{T} e-\frac{(\gamma-1)}{a}\left(\frac{1}{2}+\frac{1}{2}\|\widehat{W}\|^{2} L_{\phi}^{2}\right) e^{T}
$$

If $\left(\lambda-1+K_{p}\right)>0, a>0,(\gamma-1)>0$, so that: $a D_{t}^{\alpha} V \leq 0, \forall e, w, \widehat{W} \neq 0, e \neq 0$, is wanted to be demonstrate.

The control law is given by Eq. (19)

$$
\begin{gathered}
u_{n}=\Omega^{\dagger}\left[-\widehat{W} \Gamma\left(z\left(x_{n}(t-\tau)\right)-z\left(x_{p}(t-\tau)\right)\right)-(A+I)\left(x-x_{p}\right)+K_{p} e+K_{i} a D_{t}^{-\alpha} e\right. \\
\quad+K_{v} a D_{t}^{\alpha} e- \\
\left.\Gamma\left(\frac{1}{2}+\frac{1}{2}\|\widehat{W}\|^{2} L_{\phi}^{2}\right) e_{n}+f_{r}\left(x_{r}, u_{r}\right)-A x_{r}-\widehat{W} \Gamma_{\mathrm{z}}\left(x_{r}(t-\tau)\right)-x_{r}+x_{p}\right]
\end{gathered}
$$

Theorem: The control law Eq. (19) and the neuronal adaptation law given by Eq. (15) guarantee that the fractional tracking error converges to zero, by which the tracking of trajectories of the non-linear system is guaranteed Eq. (4).

\section{Identification of the unknown non-linear system by the neural network}

Following the procedure above, we obtain the following control law. Let $V$ be, the next candidate Lyapunov function as

$$
V=\frac{1}{2}\left(e_{p}^{T}, w^{T}\right)\left(e_{p}, w\right)^{T}+\frac{1}{2 a} \operatorname{tr}\left\{\widetilde{W}^{T} \widetilde{W}\right\}+\frac{1}{a} \int_{t-\tau}^{t}\left[\emptyset_{\sigma}^{T}(s) \widehat{W}^{T} \widehat{W} \emptyset_{\sigma}(s)\right] d s
$$

Then, (21) is reduced to

$$
a D_{t}^{\alpha} V \leq \frac{-1}{a}\left(\lambda-1+K_{p}\right)\left(e_{p}^{T}\right)\left(e_{p}\right)-\frac{(\gamma-1)}{a}\left(\frac{1}{2}+\frac{1}{2}\|\widehat{W}\|^{2} L_{\phi}^{2}\right)\left(e_{p}^{T}\right)\left(e_{p}\right)<0
$$

The previous inequality guarantees that the identification of the non-linear system is satisfied, that is, the approach error converges to zero asymptotically

$$
\begin{gathered}
u_{p}=\Omega^{\dagger}\left[\widehat{W} \Gamma z\left(x_{r}(t-\tau)\right)-\widehat{W} \Gamma\left(z\left(x_{n}(t-\tau)\right)-z\left(x_{p}(t-\tau)\right)\right)-\right. \\
(A+I)\left(x-x_{p}\right)+K_{p} e+K_{i} a D_{t}^{-\alpha} e+K_{v} a D_{t}^{\alpha} e-\Gamma\left(\frac{1}{2}+\frac{1}{2}\|\widehat{W}\|^{2} L_{\phi}^{2}\right) e_{n}- \\
\left.\Gamma\left(\frac{1}{2}+\frac{1}{2}\|\widehat{W}\|^{2} L_{\phi}^{2}\right) e_{p}+f_{r}\left(x_{r}, u_{r}\right)-f_{p}\left(x_{p}\right)+A x_{p}-A x_{r}+\widehat{W} \Gamma_{\mathrm{z}}\left(x_{p}\right)-x_{r}+x_{p}\right]
\end{gathered}
$$

\section{Simulation}

The mathematical model, which describes the movement dynamics of the bipedal robot (figure 2), is obtained using the Euler-Lagrange equations [1], [13].

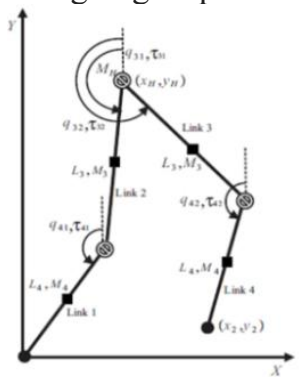

Fig. 2. Dynamic model of Biped Robot. 


$$
D(q(t) \ddot{q}(t)+C(q(t), \dot{q}(t)) \dot{q}(t)+G(q(t))=B \tau(t)
$$

where $q(t)=\left[q_{31}(t) q_{32}(t) q_{41}(t) q_{42}(t)\right]^{T}$, is the generalized coordinates vector. As usual, $D(q(t))$ is the inertia matrix, bounded and positive definite, and $C(q(t), \dot{q}(t))$ is the matrix of Coriolis and centripetal forces. $G(q(t))$ represents a matrix of gravitational effects and $B$ defines the input matrix. The vector $\tau(t)=\left[\tau_{31}(t) \tau_{32}(t) \tau_{41}(t) \tau_{42}(t)\right]^{T}$, defines the applied joint torques of the robot.

To illustrate the theoretical results obtained, we propose an example, which, as can be seen in the simulations, trajectory tracking is guaranteed and the neural network is described by:

$a D_{t}^{\alpha} x_{p}=\mathrm{A}(x)+\mathrm{W}^{*} \Gamma_{\mathrm{z}}(x(t-\tau))+\Omega_{\mathrm{u}}$, with $\tau=25 \mathrm{sec}, A=-20 I, I \in \Re^{4 x 4}$, and, $\mathrm{W}^{*}$ is estimated using the learning law given in (15).

$\Gamma_{\mathrm{z}}(x(t-\tau))=\left(\tanh \left(x_{1}(t-\tau)\right), \tanh \left(x_{2}(t-\tau)\right), \ldots, \tanh \left(x_{n}(t-\tau)\right)\right)^{\mathrm{T}} \quad, \quad \Omega=$ $\left(\begin{array}{cccc}0 & 0 & 1 & 0 \\ 0 & 0 & 0 & 1\end{array}\right)^{\mathrm{T}}$ and the $u$ is obtained using (22), and the reference signal that they have to follow, both the non-linear system and the neural network is given by the Duffing equation [14].

$$
\begin{gathered}
\ddot{x}-x+x^{3}=0.114 \cos (1.1 t): x(0)=1, \dot{x}(0)=0.114 \\
\frac{x(t)}{d t}=y(t) \\
\frac{y(t)}{d t}=x(t)-x^{3}(t)-\alpha y(t)+\delta \cos (\omega t)
\end{gathered}
$$

Here, the conventional derivatives are replaced by the fractional derivatives as follows:

$$
\begin{gathered}
a D_{t}^{\alpha} x(t)=y(t) \\
a D_{t}^{\alpha} x(t)=x(t)-x^{3}(t)-\alpha y(t)+\delta \cos (\omega t)
\end{gathered}
$$

where $\alpha, \omega, \delta$, are the parameters of the Duffing differential equation, which we will use as a reference trajectory, that the non-linear system and the neural network have to follow.

As can be seen in figures 4 and 5, the tracking of trajectories in the states of the system are performed with satisfaction, while figure 3 shows the plane phase of the same fractional order differential equation. Figures 6,7 show the torques applied to the ends of the bipedal robot. Parameter values of the fractional order, alpha $(0.0001)$ and beta $(0.0001)$ are included.

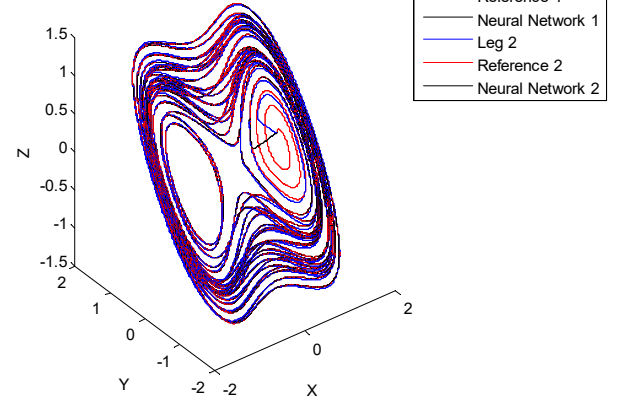

Fig. 3. A phase space trajectory of Duffing equation.

$$
\alpha=0.001, \beta=0.001
$$

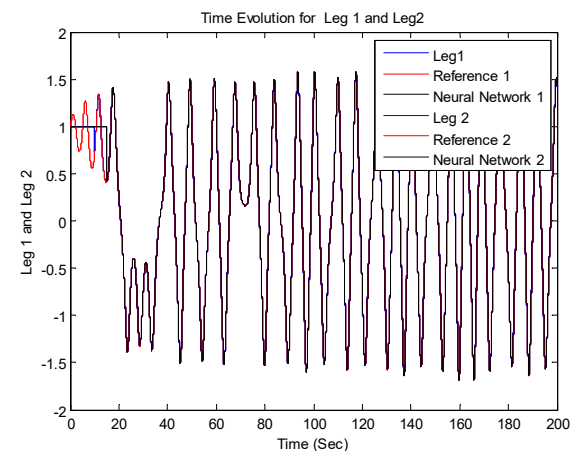

Fig. 4. Time evolution for the angular Position Leg 1 and Leg 2 (rad) of Link 1. 


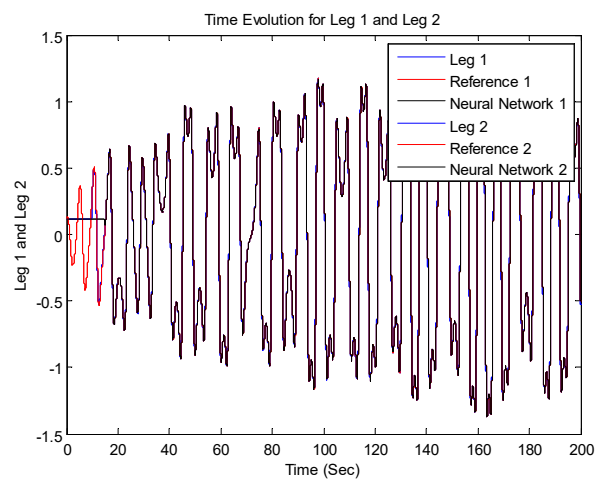

Fig. 5. Time evolution for the angular Position Leg 1 and and Leg 2(rad) of Link 2.

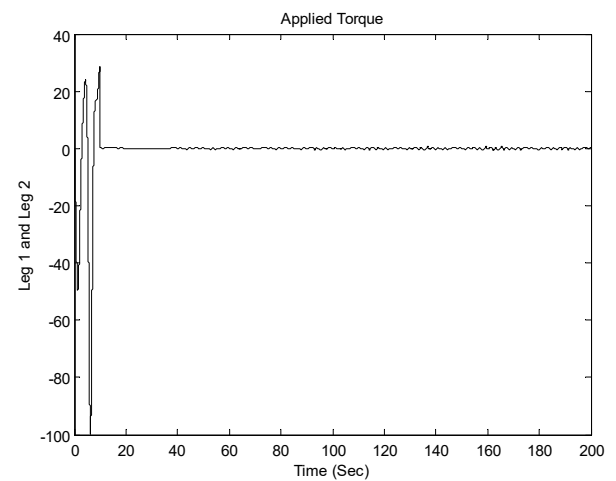

Fig. 6. Torque (Nm) applied to Leg 1 and Leg 2 of Link 1. Applied Torque

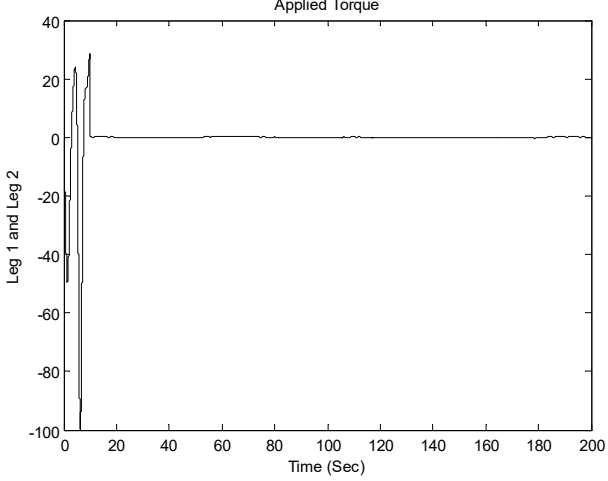

Fig. 7. Torque applied to Leg 1 of Link 2.

\section{Conclusions}

In this research work, conditions have been obtained in the parameters of the adaptive recurrent neural network, as well as laws of control and laws of neuronal adaptation, which, together, guarantee that the tracking error of trajectories between the non-linear system and the reference signal converges asymptotically to zero, so that trajectory tracking is develops with satisfaction.

\section{Acknowledgements}

Authors thank, Mexican National Science and Technology Council, (CONACyT), Mexico, to theAutonomous University of Nuevo Leon, Dynamical Systems Group of the Department of Physical and Mathematical Sciences, (FCFM-UANL), Mexico.

\section{References}

1. Design and Real-Time Control of a 4-DOF Biped Robot, Jose Alejandro Vazquez and Martin Velasco-Villa, International Journal of Advanced Robotic Systems, Intech, 2013.

2. Nonlinear Dynamics and Chaos in Fractional-Order Hopfield Neural Networks with Delay, Xia Huang, Zhen Wang, and Yuxia Li, Hindawi Publishing Corporation, 
Advances in Mathematical Physics, Volume 2013, Article ID 657245, 9 pages, http://dx.doi.org/10.1155/2013/657245

3. Stability analysis of fractional-order Hopfield neural networks with discontinuous activation functions, Shuo Zhang, Yongguang Yu, Qing Wang, Neurocomputing 171 (2016) 1075-1084, www.elsevier.com/locate/neucom.

4. Integer \& Fractional Order PID Controller for Fractional Order Subsystems of AUV, Mrs. Sneha D. Joshi, Dr. D. B. Talange, 2013 IEEE Symposium on Industrial Electronics \& Applications (ISIEA2013), September 22-25, 2013, Kuching, Malaysia.

5. R. Kelly, R. E. Haber, R.E. Haber Guerra and Fernando Reyes, "Lyapunov Stable Control of Robot Manipulators: a Fuzzy Self-TuningProcedure", Intelligent Automation and Soft Computing, Vol. 5, No. 4, pp. 313-326, 1999.

6. Arturo Rojas.Moreno, Victor Jara.Sandoval, Fractional Order PD and PID Position Control of an Angular Manipulator of 3DOF, arojas@utec.edu.pe, jaravictor2000@yahoo.com.

7. Fractional Versions of the Fundamental Theorem of Calculus, Eliana Contharteze Grigoletto, Edmundo Capelas de Oliveira, Applied Mathematics, 2013, 4, 23-33, Scientific Research, http://dx.doi.org/10.4236/am.2013.47A006, (http://www.scirp.org/journal/am).

8. Fractional order Lyapunov stability theorem and its applications in synchronization of complex dynamical networks, Diyi Chen, Runfan Zhang, Xinzhi Liu, Xiaoyi Ma, Commun Nonlinear Sci Numer Simulat 19 (2014) 4105-4121, www.elsevier.com/locate/cnsns

9. Disturbance Rejection for Fractional-Order Time-Delay Systems, Hai-Peng Jiang and Yong-Qiang Liu, Hindawi Publishing Corporation, Mathematical Problems in Engineering, Volume 2016, Article ID 1316046, 8 pages, http://dx.doi.org/10.1155/2016/1316046

10. Rovitahkis G. A. and M. A. Christodoulou, Adaptive Control with Recurrent HighOrder Neural Networks, Springer Verlang, New York,USA, 2000.

11. Petros A. Ioannou, Jing Sun, "Robust Adaptive Control", PTR Prentice-Hall, Upper Saddle River, NJ 07458. ISBN 0-13-439100-4.

12. Adaptive Synchronization of Fractional Neural Networks with Unknown Parameters and Time Delays, Weiyuan Ma, Changpin Li, Yujiang $\mathrm{Wu}$ and Yongqing $\mathrm{Wu}$, Entropy 2014, 16, 6286-6299; doi:10.3390/e16126286, ISSN 1099-4300.

13. Mark W. Spong and M. Vidyasagar, Robot Dynamics and Control. John Wiler and Sons, USA, 1989.

14. Ivo Petras, Nonlinear Physical Science, Fractional-Order Nonlinear Systems, Modeling, Analysis and Simulation, Springer,Springer Heidelberg Dordrecht London New York, ISSN 1867-8440, ISBN 978-3-642-18100-9. 